\title{
Tracking Primary Thermalization Events in Graphene with Photoemission at Extreme Time Scales
}

\author{
I. Gierz, ${ }^{1, *}$ F. Calegari, ${ }^{1,2}$ S. Aeschlimann, ${ }^{1}$ M. Chávez Cervantes, ${ }^{1}$ C. Cacho, ${ }^{3}$ R. T. Chapman, ${ }^{3}$ \\ E. Springate, ${ }^{3}$ S. Link, ${ }^{4}$ U. Starke, ${ }^{4}$ C. R. Ast,${ }^{4}$ and A. Cavalleri ${ }^{1,5}$ \\ ${ }^{1}$ Max Planck Institute for the Structure and Dynamics of Matter, Center for Free Electron Laser Science, 22761 Hamburg, Germany \\ ${ }^{2}$ Institute for Photonics and Nanotechnologies, IFN-CNR, 20133 Milano, Italy \\ ${ }^{3}$ Central Laser Facility, STFC Rutherford Appleton Laboratory, OX11 OQX Harwell, United Kingdom \\ ${ }^{4}$ Max Planck Institute for Solid State Research, 70569 Stuttgart, Germany \\ ${ }^{5}$ Department of Physics, Clarendon Laboratory, University of Oxford, OX1 3PU Oxford, United Kingdom
}

(Received 3 June 2015; published 21 August 2015)

\begin{abstract}
Direct and inverse Auger scattering are amongst the primary processes that mediate the thermalization of hot carriers in semiconductors. These two processes involve the annihilation or generation of an electronhole pair by exchanging energy with a third carrier, which is either accelerated or decelerated. Inverse Auger scattering is generally suppressed, as the decelerated carriers must have excess energies higher than the band gap itself. In graphene, which is gapless, inverse Auger scattering is, instead, predicted to be dominant at the earliest time delays. Here, $<8$ fs extreme-ultraviolet pulses are used to detect this imbalance, tracking both the number of excited electrons and their kinetic energy with time-and angleresolved photoemission spectroscopy. Over a time window of approximately $25 \mathrm{fs}$ after absorption of the pump pulse, we observe an increase in conduction band carrier density and a simultaneous decrease of the average carrier kinetic energy, revealing that relaxation is in fact dominated by inverse Auger scattering. Measurements of carrier scattering at extreme time scales by photoemission will serve as a guide to ultrafast control of electronic properties in solids for petahertz electronics.
\end{abstract}

DOI: 10.1103/PhysRevLett.115.086803

PACS numbers: 73.22.Pr, 78.47.J-, 79.60.-i

The dynamics of prethermal Dirac carriers in graphene are expected to host interesting and unconventional phenomena. Microscopic simulations [1,2] predict that different scattering events mediated by the Coulomb interaction may contribute to the ultrafast redistribution of the photoexcited electrons (see Fig. 1). For example, these theories imply that inverse Auger scattering (also known as impact ionization) may dominate at early times.

This can be understood as follows [1,2]. As shown in Fig. 1, for a nonequilibrium distribution with holes in the valence band at $E=E_{D}-\frac{1}{2} \hbar \omega_{\text {pump }}$ and electrons in the conduction band at $E=E_{D}+\frac{1}{2} \hbar \omega_{\text {pump }}$ (where $E_{D}$ and $\hbar \omega_{\text {pump }}$ are the Dirac point and pump photon energy, respectively) in undoped graphene, the recombination of electron-hole pairs required for Auger heating is strongly suppressed due to the lack of holes at the top of the valence band. On the other hand, the available phase space for the inverse process (impact ionization), where the excess energy of an electron high in the conduction band is used to generate secondary electron-hole pairs, is large. Therefore, impact ionization is believed to dominate over Auger heating for as long as it takes to establish a thermalized electronic distribution. The resulting carrier multiplication, for which the absorption of a single photon may generate multiple electron-hole pairs, has raised interest for possible applications in photovoltaic devices.
However, these theoretical arguments apply only for undoped graphene and low excitation fluences (few $\left.\mu \mathrm{J} / \mathrm{cm}^{2}\right)$. At high pump fluences, the balance between impact ionization and Auger heating is predicted to be reestablished within a few tens of femtoseconds, reducing the carrier multiplication factor considerably [1,2]. Furthermore, real graphene samples typically rest on a

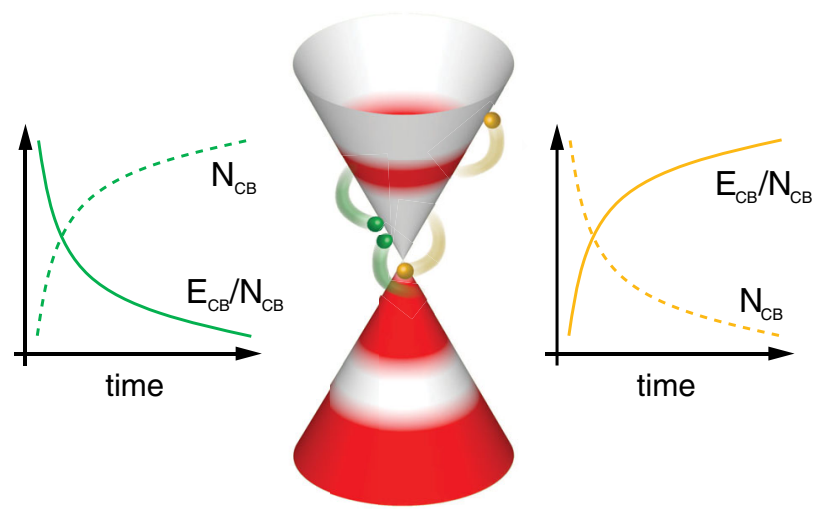

FIG. 1 (color online). Different Coulomb-interaction-mediated scattering processes in photoexcited graphene: impact ionization (green) and Auger heating (yellow). These processes can be identified experimentally by comparing the total number of electrons inside the conduction band (CB), $N_{\mathrm{CB}}$, with the average kinetic energy of electrons inside the conduction band, $E_{\mathrm{CB}} / N_{\mathrm{CB}}$. Occupied states are shown in red, empty states are shown in white. 
substrate resulting in a non-negligible doping of the graphene layer. The presence of either hole or electron doping will shift the relative importance of impact ionization and Auger heating, suppressing carrier multiplication.

Previous optical experiments deduced transient electron distribution functions by comparing differential transmission, reflectivity, or absorption data to model calculations. In this way, high-temporal-resolution experiments estimated an electronic thermalization time between 13 and 50 fs $[3,4]$. Furthermore, indirect evidence for carrier multiplication with $\sim 200 \mathrm{fs}$ pulses in the low fluence regime $\left(<30 \mu \mathrm{J} / \mathrm{cm}^{2}\right)$ was obtained by comparing the number of absorbed photons to the number of electron-hole pairs [5] with some indications already in [3].

Photoemission techniques, which directly measure electron numbers as a function of energy and momentum, are ideally suited for a direct visualization of Auger scattering. Previous time- and angle-resolved photoemission spectroscopy (TR-ARPES) experiments were performed at pump fluences on the order of $\mathrm{mJ} / \mathrm{cm}^{2}$ and, thus, short time scales for the initial thermalization. The temporal resolution of $\geq 30$ fs was then insufficient to resolve prethermal carrier distributions [6-13].

Here, we use TR-ARPES with $\leq 10$ fs pump and probe pulses to access charge carrier dynamics in photoexcited graphene. The experiments were performed using the Materials Science end station at the Artemis user facility at the Rutherford Appleton Laboratory in Harwell, United Kingdom. The setup consists of a titanium:sapphire amplifier $(\hbar \omega=1.55 \mathrm{eV})$ operating at $1 \mathrm{kHz}$ with $30 \mathrm{fs}$ pulse duration. $1 \mathrm{~mJ}$ of energy was compressed down to 8 fs with a hollow-core fiber filled with 1.4 bar of neon operated with a pressure gradient and followed by ten chirped mirrors. Pulse compression resulted in a slight blue shift of the spectrum. $400 \mu \mathrm{J}$ of energy were used for high harmonics generation (HHG) in argon, $20 \mathrm{~mJ} / \mathrm{cm}^{2}$ were used to excite the sample. Because of the longer path in air, two additional chirped mirrors were added to the pump path resulting in a pump pulse duration of $10 \mathrm{fs}$. All pulse durations were measured using second-harmonic frequency-resolved optical gating. Out of the broad HHG spectrum, one particular energy, $\hbar \omega_{\text {probe }}=30 \mathrm{eV}$, is selected by a time-preserving grating monochromator [14] and used as a probe pulse for photoemission. For the present investigation, two different gratings with different dispersion (G300 with 300 grooves per mm and G60 with 60 grooves per $\mathrm{mm}$ ) have been used for wavelength selection, optimizing either time or energy resolution [14]. Both pump and probe pulses were $s$ polarized, with the electric field vector in the plane of the graphene sample perpendicular to the $\Gamma K$ direction.

Quasifreestanding epitaxial graphene samples on silicon carbide were grown as previously described in [15]. Prior to graphene growth the silicon carbide substrates were etched in a hydrogen atmosphere to remove scratches from mechanical polishing. In a second step, the substrates were annealed in an argon atmosphere resulting in the growth of one carbon monolayer on the silicon-terminated face of the substrate. This carbon monolayer was subsequently decoupled from the substrate by hydrogen intercalation and characterized by static ARPES measurements (see Supplemental Material [16]). The resulting graphene samples are lightly hole doped with the Dirac point $\sim 200 \mathrm{meV}$ above the equilibrium chemical potential. The samples were transported to the Artemis user facility under ambient conditions, reinserted into ultrahigh vacuum, and cleaned by a mild annealing, recovering the original band structure.

In Fig. 2(a), we show TR-ARPES snapshots of graphene's linear $\pi$ bands along the $\Gamma K$ direction for selected pump-probe time delays across the rising edge of the pumpprobe signal. Because of the $s$ polarization of the probe pulse [21] and photoelectron interference effects [22], only the right-hand branch of the Dirac cone was visible in this geometry. These snapshots were recorded with a temporal resolution of $14 \mathrm{fs}$ and an energy resolution of $500 \mathrm{meV}$ (for details, see Supplemental Material [16]) achieved by using a monochromator grating with 300 grooves per millimeter (G300) [14]. The optical matrix element describing the absorption of the pump photon is anisotropic with nodes along the direction of the pump polarization (in this case, perpendicular to the $\Gamma K$ direction) and maxima in the direction perpendicular to the pump polarization (in this case $\Gamma K$ ) [23-25]. We probed the response of the electronic structure along the $\Gamma K$ direction where the effect of the pump pulse is the strongest.

The pump-induced changes of the photocurrent are plotted in Fig. 2(b). A loss (gain) of electrons below (above) the equilibrium chemical potential, which is used here as zero-energy reference, is observed.

From the snapshots in Fig. 2(a), transient electron distribution functions were extracted and compared to FermiDirac (FD) distributions in Fig. 2(c). For that purpose, we took lineouts at constant angle (energy distribution curves) from the raw data, determined their integrated intensity within the full width at half maximum (FWHM) of the peak and attributed the resulting number to the energy of the peak position (for details, see Supplemental Material [16]). At negative delays, immediately before arrival of the pump pulse [-10 fs in Fig. 2(c)], the distribution follows a FD distribution, indicating the presence of a completely thermalized electron gas. Near zero time delay, a shoulder develops in the conduction band above $E_{D}=200 \mathrm{meV}$. This shoulder cannot be fitted with a FD distribution. The residual weight between the experimental distribution function [red line in Fig. 2(c)] and the FD fit [dashed black line in Fig. 2(c)] is taken as proportional to the number of nonthermal carriers [NTCs, blue shaded area in Fig. 2(c)]. We find that the number of NTCs keeps increasing until the peak of the pump-probe signal is 

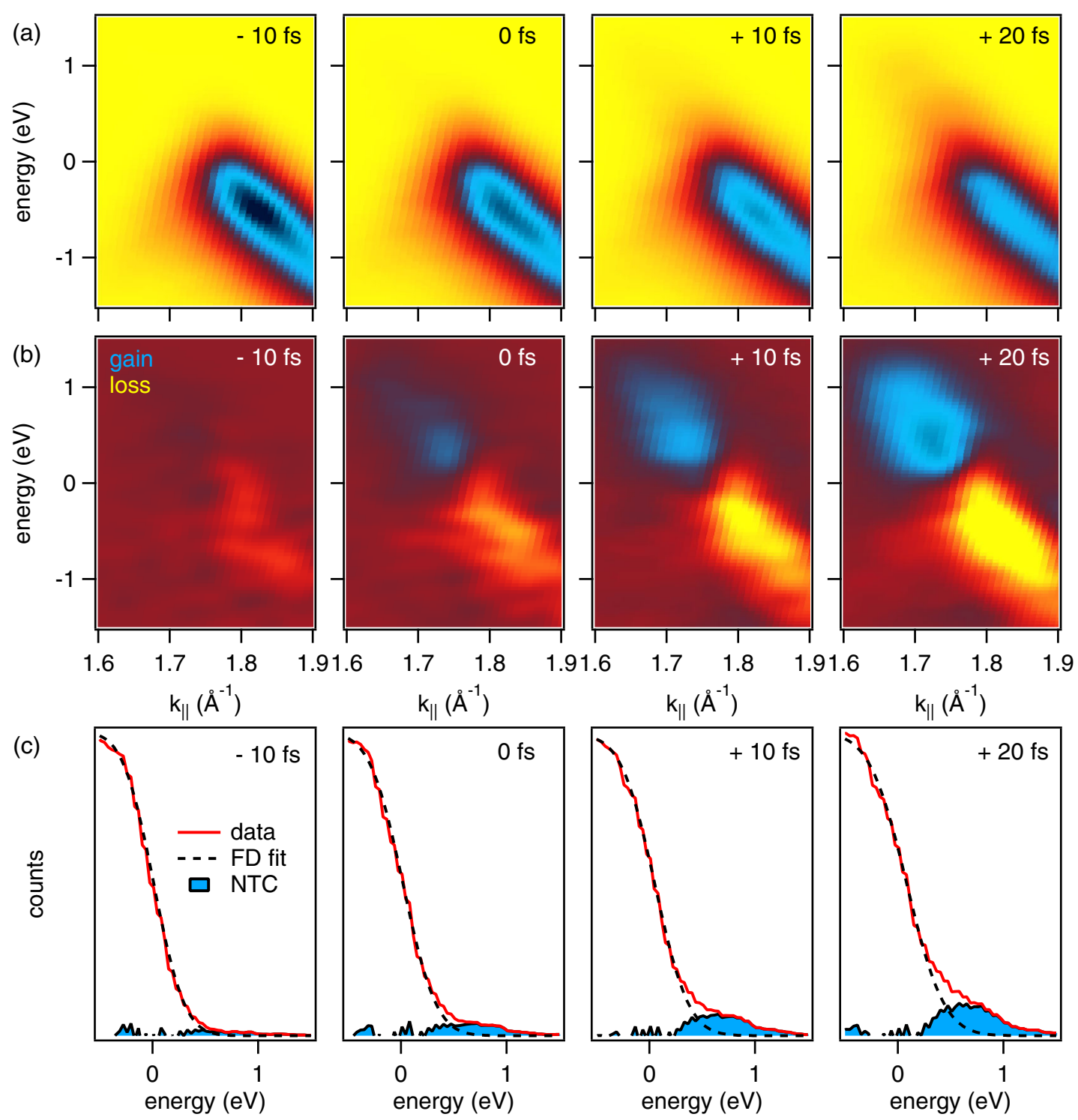

FIG. 2 (color online). TR-ARPES measurements. (a) Snapshots for different pump-probe time delays. (b) Corresponding pumpinduced changes of the photocurrent. The data in panels (a) and (b) have been smoothed. (c) Electronic distribution functions (red line) together with Fermi-Dirac fits (black dashed line) revealing the presence of nonthermalized carriers (blue). The $y$-axis scale in (c) is linear. The measurements presented in this figure were carried out using the high-energy-resolution grating G300 (for details, see text).

reached at a time delay of $\sim 20$ fs. These NTCs can be understood as a precursor of the inverted carrier population observed in previous studies with longer pump pulses at slightly smaller photon energies [7,12,26].

Higher-temporal-resolution measurements were performed with a second monochromator grating with 60 grooves per millimeter (G60), delivering a temporal resolution of $8 \mathrm{fs}$ and an energy resolution of $800 \mathrm{meV}$ (see Supplemental Material [16]). The number of carriers in the conduction band at $E>E_{D}, N_{\mathrm{CB}}$, and their average kinetic energy, $E_{\mathrm{CB}} / N_{\mathrm{CB}}$, were determined directly from the raw data (see Supplemental Material [16]) and displayed in Fig. 3. For about 25 fs around zero pumpprobe time delay, the average kinetic energy $E_{\mathrm{CB}} / N_{\mathrm{CB}}$ was observed to decrease while the number of carriers $N_{\mathrm{CB}}$ kept increasing, indicating impact ionization.

From these measurements, the following scenario can be envisaged. Impact ionization is the primary scattering mechanism during the first $\sim 25 \mathrm{fs}$, accumulating carriers at the bottom of the conduction band and establishing a precursor of the population inversion observed previously $[7,12,26]$. This state, then, likely decays through Auger heating and electron-phonon scattering within $\sim 100$ fs, reestablishing a single Fermi Dirac distribution [7]. We note that the temporal evolution of the electronic temperature and the decay time of the nonthermal carriers found in our experiment (see Supplemental Material [16]) further substantiate this interpretation. 


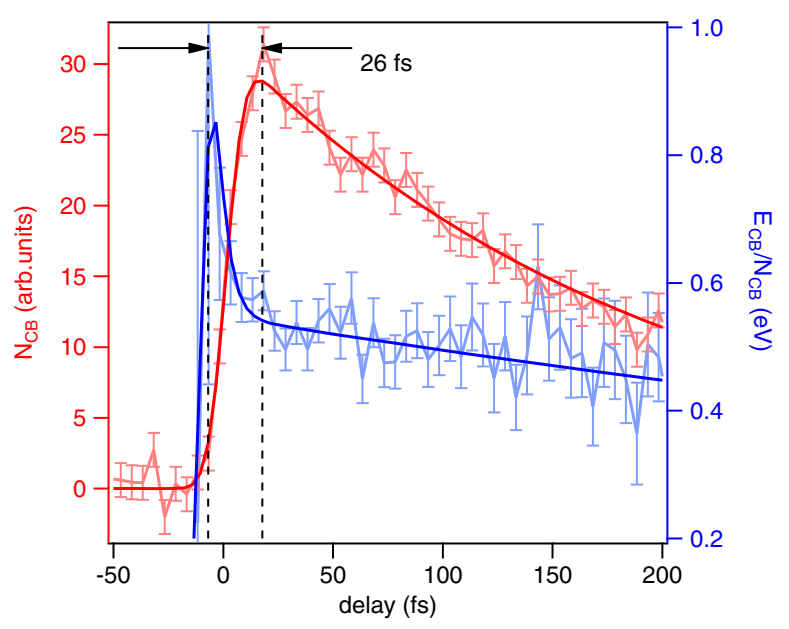

FIG. 3 (color online). Direct evidence for impact ionization. Comparison between the temporal evolution of the total number of carriers inside the conduction band $\left(N_{\mathrm{CB}}\right.$, light red) and the temporal evolution of their average kinetic energy $\left(E_{\mathrm{CB}} / N_{\mathrm{CB}}\right.$, light blue). Around zero time delay, $E_{\mathrm{CB}} / N_{\mathrm{CB}}$ already decreases while $N_{\mathrm{CB}}$ keeps increasing, indicating impact ionization. Dark red and blue lines are fits to the data that serve as guides to the eye. The fitting function consists of an error function to describe the rising edge plus a single $\left(N_{\mathrm{CB}}\right)$ or double exponential decay $\left(E_{\mathrm{CB}} / N_{\mathrm{CB}}\right)$. The measurements presented in this figure were carried out using the low-energy-resolution grating G60 (for details, see text). Error bars represent the standard deviation.

In summary, we have used TR-ARPES with $\leq 10$ fs pulses to identify the primary scattering events that result in the rapid thermalization of photoexcited electron-hole pairs in graphene. By comparing the number of electrons inside the conduction band with their average kinetic energy, we find that impact ionization is the predominant scattering channel within the first $\sim 25$ fs also in doped graphene at high fluence. Whether the observed carrier multiplication can be exploited for solar cell applications remains questionable, as the absence of a band gap in graphene makes charge separation difficult. Nevertheless, the ultrafast dynamics of photoexcited electron-hole pairs observed here will be of use for the design of new electronic and optoelectronic devices operating at petahertz rates [27] based on graphene and other materials.

We thank A.S. Wyatt, O. Alexander, and P. Rice for technical support during the beam time, and J. Harms for support with the figures. This work was supported by the German Research Foundation (DFG) in the framework of the Priority Program 1459 'Graphene.' Access to the Artemis facility at the Rutherford Appleton Laboratory was funded by STFC.

*Isabella.Gierz@mpsd.mpg.de

[1] T. Winzer, A. Knorr, and E. Malic, Nano Lett. 10, 4839 (2010).
[2] T. Winzer and E. Malic, Phys. Rev. B 85, 241404(R) (2012).

[3] D. Brida, A. Tomadin, C. Manzoni, Y. J. Kim, A. Lombardo, S. Milana, R. R. Nair, K. S. Novoselov, A. C. Ferrari, G. Cerullo, and M. Polini, Nat. Commun. 4, 1987 (2013).

[4] M. Breusing, S. Kuehn, T. Winzer, E. Malic, F. Milde, N. Severin, J. P. Rabe, C. Ropers, A. Knorr, and T. Elsaesser, Phys. Rev. B 83, 153410 (2011).

[5] T. Plötzing, T. Winzer, E. Malic, D. Neumaier, A. Knorr, and H. Kurz, Nano Lett. 14, 5371 (2014).

[6] J. C. Johannsen, S. Ulstrup, F. Cilento, A. Crepaldi, M. Zacchigna, C. Cacho, I. C. Edmond Turcu, E. Springate, F. Fromm, C. Raidel, T. Seyller, F. Parmigiani, M. Grioni, and P. Hofmann, Phys. Rev. Lett. 111, 027403 (2013).

[7] I. Gierz, J. C. Petersen, M. Mitrano, C. Cacho, I. C. E. Turcu, E. Springate, A. Stöhr, A. Köhler, U. Starke, and A. Cavalleri, Nat. Mater. 12, 1119 (2013).

[8] I. Gierz, S. Link, U. Starke, and A. Cavalleri, Faraday Discuss. 171, 311 (2014).

[9] S. Ulstrup, J. C. Johannsen, F. Cilento, J. A. Miwa, A. Crepaldi, M. Zacchigna, C. Cacho, R. Chapman, E. Springate, S. Mammadov, F. Fromm, C. Raidel, T. Seyller, F. Parmigiani, M. Grioni, P. D. C. King, and P. Hofmann, Phys. Rev. Lett. 112, 257401 (2014).

[10] J. C. Johannsen, S. Ulstrup, A. Crepaldi, F. Cilento, M. Zacchigna, J. A. Miwa, C. Cacho, R. T. Chapman, E. Springate, F. Fromm, C. Raidel, T. Seyller, P. D. C. King, F. Parmigiani, M. Grioni, and P. Hofmann, Nano Lett. 15, 326 (2015).

[11] S. Ulstrup, J. C. Johannsen, A. Crepaldi, F. Cilento, M. Zacchigna, C. Cacho, R. T. Chapman, E. Springate, F. Fromm, C. Raidel, T. Seyller, F. Parmigiani, M. Grioni, and P. Hofmann, J. Phys. Condens. Matter 27, 164206 (2015).

[12] I. Gierz, M. Mitrano, J. C. Petersen, C. Cacho, I. C. E. Turcu, E. Springate, A. Stöhr, A. Köhler, U. Starke, and A. Cavalleri, J. Phys. Condens. Matter 27, 164204 (2015).

[13] I. Gierz, M. Mitrano, H. Bromberger, C. Cacho, R. Chapman, E. Springate, S. Link, U. Starke, B. Sachs, M. Eckstein, T. O. Wehling, M. I. Katsnelson, A. Lichtenstein, and A. Cavalleri, Phys. Rev. Lett. 114, 125503 (2015).

[14] F. Frassetto, C. Cacho, C. A. Froud, I. C. E. Turcu, P. Villoresi, W. A. Bryan, E. Springate, and L. Poletto, Opt. Express 19, 19169 (2011).

[15] C. Riedl, C. Coletti, T. Iwasaki, A. A. Zakharov, and U. Starke, Phys. Rev. Lett. 103, 246804 (2009).

[16] See Supplemental Material at http://link.aps.org/ supplemental/10.1103/PhysRevLett.115.086803, which includes Refs. [17-20], for experimental methods, sample preparation, and additional experimental data.

[17] S. Ulstrup, J. C. Johannsen, M. Grioni, and P. Hofmann, Rev. Sci. Instrum. 85, 013907 (2014).

[18] H. Yan, D. Song, K. F. Mak, I. Chatzakis, J. Maultzsch, and T. F. Heinz, Phys. Rev. B 80, 121403(R) (2009).

[19] K. Kang, D. Abdula, D. G. Cahill, and M. Shim, Phys. Rev. B 81, 165405 (2010).

[20] J. C. W. Song, M. Y. Reizer, and L. S. Levitov, Phys. Rev. Lett. 109, 106602 (2012).

[21] I. Gierz, J. Henk, H. Höchst, C. R. Ast, and K. Kern, Phys. Rev. B 83, 121408(R) (2011).

[22] E. L. Shirley, L. J. Terminello, A. Santoni, and F. J. Himpsel, Phys. Rev. B 51, 13614 (1995). 
[23] E. Malic, T. Winzer, E. Bobkin, and A. Knorr, Phys. Rev. B 84, 205406 (2011).

[24] M. Mittendorff, T. Winzer, E. Malic, A. Knorr, C. Berger, W. A. de Heer, H. Schneider, M. Helm, and S. Winnerl, Nano Lett. 14, 1504 (2014).

[25] X.-Q. Yan, J. Yao, Z.-B. Liu, X. Zhao, X.-D. Chen, C. Gao, W. Xin, Y. Chen, and J.-G. Tian, Phys. Rev. B 90, 134308 (2014).
[26] T. Li, L. Luo, M. Hupalo, J. Zhang, M. C. Tringides, J. Schmalian, and J. Wang, Phys. Rev. Lett. 108, 167401 (2012).

[27] M. Schultze, E. M. Bothschafter, A. Sommer, S. Holzner, W. Schweinberger, M. Fiess, M. Hofstetter, R. Kienberger, V. Apalkov, V. S. Yakovlev, M. I. Stockman, and F. Krausz, Nature (London) 493, 75 (2013). 